\title{
Chapter 19 \\ Verification of the Repeatability of Soil \\ Liquefaction Centrifuge Testing at \\ Rensselaer
}

\author{
Evangelia Korre, Tarek Abdoun, and Mourad Zeghal
}

\begin{abstract}
The Liquefaction Experiments and Analysis Projects (LEAP) is an international effort to use experimental data from physical modeling at different (international) centrifuge facilities to validate soil liquefaction numerical models and analysis tools. The goals of LEAP-2017 experimental efforts are to assess the repeatability potential at each facility, the reproducibility of centrifuge tests among different facilities, and the sensitivity of the experimental results to variation of testing parameters and conditions. A number of tests of the same (sloping deposit) centrifuge model were repeated at Rensselaer Polytechnic Institute in 2015 and 2017. This paper focuses on two specific tests to assess and demonstrate repeatability at this facility.
\end{abstract}

\subsection{Introduction}

The Liquefaction Experiments and Analysis Projects (LEAP) is an international effort to (1) investigate the dynamic response and liquefaction of a number of soil systems by means of centrifuge testing of small-scale physical models, and (2) use the experimental data to validate soil liquefaction numerical models and analysis tools.

In 2015, a LEAP was undertaken using a small-scale model of a sloping deposit. A number of tests were conducted at Cambridge University, Kyoto University, NCU of Taiwan, Rensselaer Polytechnic Institute (RPI), UC Davis, and Zhejiang University (Kutter et al. 2017, 2019). The data provided by these tests were used in a first validation exercise (Manzari et al. 2018). The LEAP 2017 expands and builds on the 2016 results and outcomes. Tests of the same sloping deposit were repeated in China (Zhejiang University), France (Institut français des sciences et technologies des transports, de l'aménagement et des réseaux), Japan (Ehime University and Kyoto University), Korea (KAIST University), Taiwan (National Taiwan University, UK (Cambridge University), and USA (UC Davis and Rensselaer). The tests are aimed

\footnotetext{
E. Korre · T. Abdoun · M. Zeghal $(\bowtie)$

Department of Civil and Environmental Engineering, Rensselaer Polytechnic Institute, Troy,

NY, USA

e-mail: zeghal@rpi.edu
} 
at assessing the repeatability, reproducibility, and sensitivity of experimental results to variability in testing conditions among the different facilities.

A total of five tests of the sloping deposit were conducted at RPI. The first two tests (referred to as 2015_RPI01 and 2015_RPI02) were executed in 2015 (Kokkali et al. 2018; Abdoun et al. 2018). The LEAP 2017 three tests were aimed at repeating the LEAP 2015 tests and also assessing the soil system response to variations in input motion (i.e., presence of a $3 \mathrm{~Hz}$ component) and deposit relative density. Specifically, the first of this series (referred to as 2017_RPI01) was simply a repetition of the first test of 2015, and was conducted according to the same specification by two different researchers. This paper presents the 2017_RPI01 test and investigates the level of repeatability with the initial test performed in 2015 (2015_RPI01). The other LEAP 2017 tests will be published elsewhere.

\subsection{Experimental Setup}

\subsubsection{Dry Model Preparation}

The dry 2017_RPI01 model was constructed according to the project specifications (Kutter et al. 2017) and following the methodology of 2015_RPI01, as described by Kokkali et al. (2018). A stratum of dry Ottawa F-65 sand (acquired from U. S. Silica) was deposited to form the specified 5-degree slope (shown in Fig. 19.1). The deposition was performed using dry pluviation in a rigid container made of aluminum and Plexiglas (Fig. 19.1). The series of experiments were all performed at a centrifugal level of $23 \mathrm{~g}$. Hereafter, all experimental results are presented in prototype scale.

Pluviation was performed manually from a specific height and at constant (lateral) velocity. This height was determined after careful calibration of the utilized pluviator and was maintained throughout the entire process of model building. The slope surface was not curved as the model longitudinal direction and direction of shaking are parallel to the Rensselaer centrifuge axis of rotation (also, curving was deemed not necessary in the transverse direction in view of the relatively small model dimension in this direction).

Fig. 19.1 The rigid container utilized in the tests conducted at Rensselaer

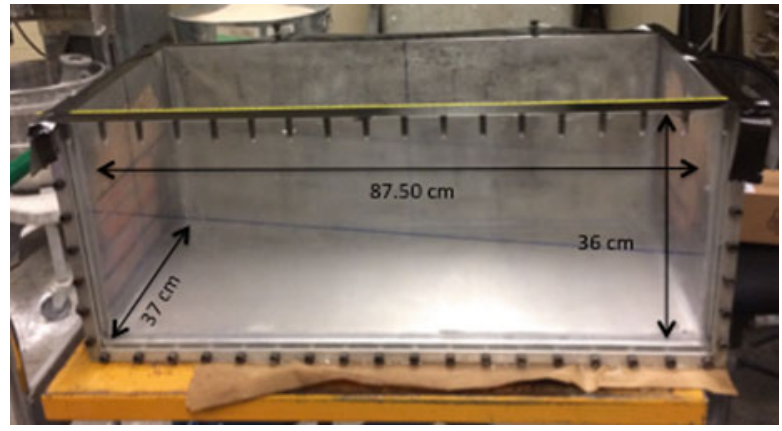


Fig. 19.2 Plan view of the box and grid used to measure the achieved mass density (dimensions in model scale)
Fig. 19.3 Mass density per horizontal layers
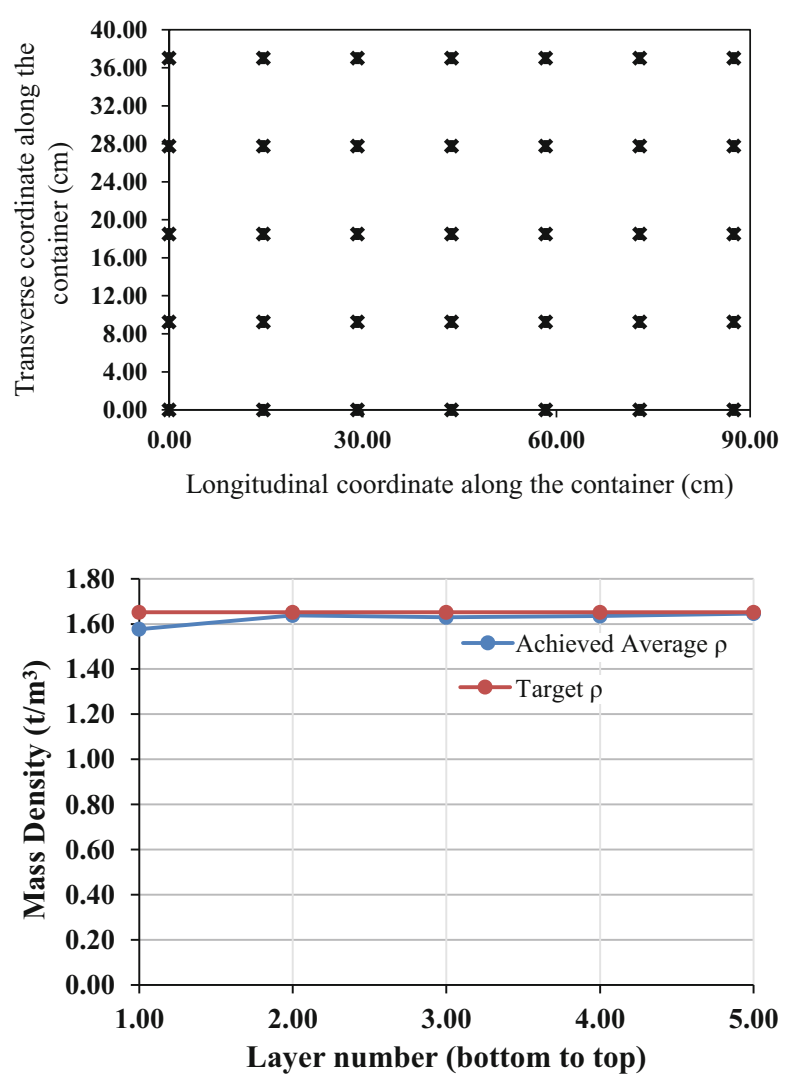

The specified target mass density of the LEAP 2015 and 2017 models was $1651 \mathrm{~kg} / \mathrm{m}^{3}$, corresponding to a relative density of $64-68 \%$ depending on which values of maximum and minimum density are assumed (Carey et al. 2019). To confirm the uniformity of the achieved deposit density, detailed mass and height measurements were taken on a grid (as shown in Fig. 19.2) at five levels along the deposit height. The measurements per layer (of about $1 \mathrm{~m}$ in thickness) are presented in Fig. 19.3 (showing the achieved and target mass densities). The first layer shows a slight discrepancy from the target. The conditions at the box boundary and the curvature around the edges (due to the applied silicone to seal the container) partly account for this discrepancy. While building the sloping surface of the deposit, it was not possible to take exact measurements until the final geometry was built. The obtained (slope) geometry is compared to the target geometry in Fig. 19.4. The achieved average density was $1.6508 \mathrm{~g} / \mathrm{cm}^{3}$, very close to the target value. 

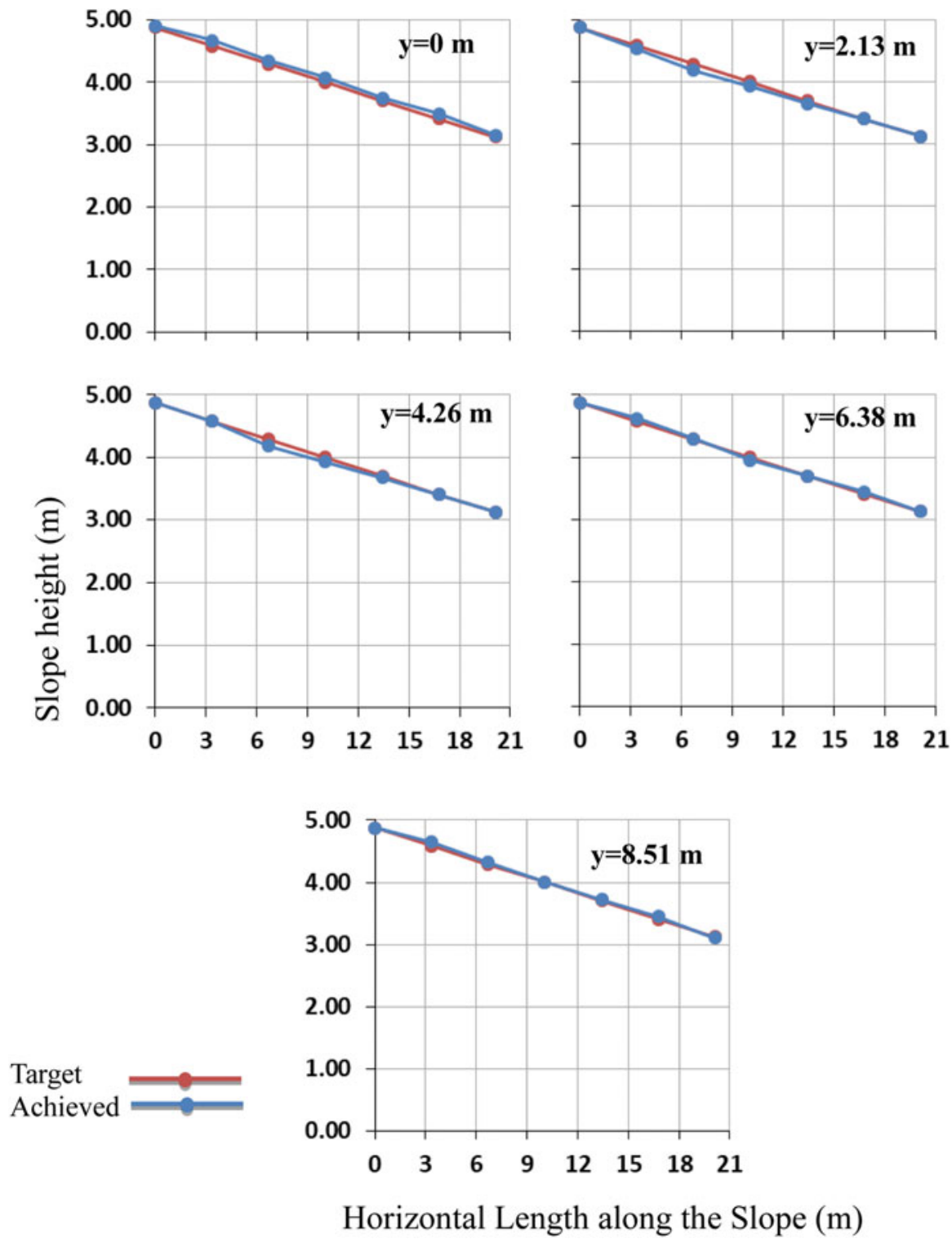

Fig. 19.4 Comparison between the target and achieved slope geometry for selected locations along the transverse $(y)$ direction

\subsubsection{Instrumentation}

The model was equipped with an extensive array of sensors that were installed according to the specified project locations (Kutter et al. 2017) while also matching the "as-built" coordinates of the sensors of 2015_RPI01. A sketch of the 


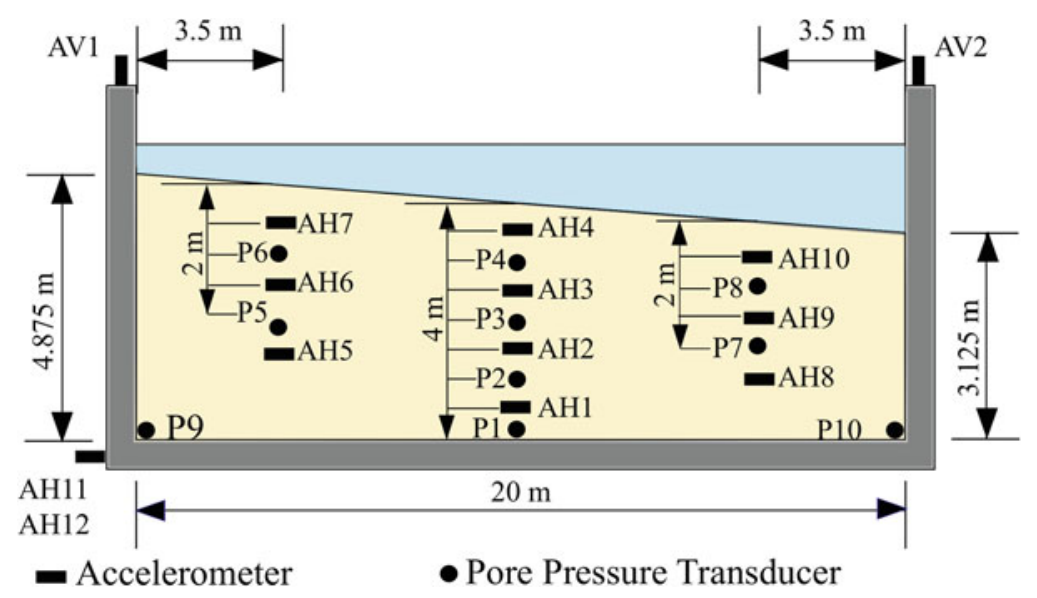

Fig. 19.5 Experimental setup showing the installed model instrumentation

experimental setup is presented in Fig. 19.5. A total of 10 accelerometers (AH1AH10) were buried within the sloping soil deposit (Fig. 19.5). Two accelerometers (AH11 and AH12) were installed at the bottom of the box to record the base longitudinal accelerations (at two opposite locations in the transverse direction), and two accelerometers (AV1 and AV2) were installed at the top of the box to record the vertical motion in the middle of the transverse side. The pore pressure was monitored by means of 10 buried pore water pressure transducers (P1-P10). In addition, zip-tie heads were inserted into the surface (of the slope) as targets which were tracked with a high-speed camera to measure the slope lateral spreading. The sensor locations inside the box (measured during construction of the model) are given in Table 19.1 in the form of $x-y-z$ coordinates. The origin of the coordinate system is at the center of the container, as prescribed in Kutter et al. (2017). Table 19.2 provides the coordinates of the grid created with the (zip-tie head) targets.

\subsubsection{Viscous Fluid Preparation and Saturation Process}

The dry model was first fixed on the centrifuge basket. Then, the container was sealed and put under vacuum. The dry model was thereafter saturated with $\mathrm{CO}_{2}$ which pushes air out of the voids. The process of vacuum and $\mathrm{CO}_{2}$ saturation was repeated twice, before starting saturation. The viscous fluid used for the saturation of the model was made with methylcellulose manufactured by Dow Chemical added at a specific ratio to the water to achieve a viscosity of $23.5 \mathrm{cp}$. 
Table 19.1 Sensor locations:

(a) accelerometers and

(b) pore water pressure transducers

\begin{tabular}{|c|c|c|c|}
\hline Sensor & $x(\mathrm{~m})$ & $y(\mathrm{~m})$ & $z(\mathrm{~m})$ \\
\hline \multicolumn{4}{|l|}{ (a) } \\
\hline AH1 & 0.2875 & -1.15 & 0.575 \\
\hline $\mathrm{AH} 2$ & 0.1725 & -1.15 & 1.495 \\
\hline AH3 & -0.0575 & -1.15 & 2.53 \\
\hline $\mathrm{AH} 4$ & -0.6325 & 1.725 & 3.45 \\
\hline AH5 & -6.6125 & -0.575 & 2.484 \\
\hline AH6 & -6.1525 & 1.035 & 3.404 \\
\hline $\mathrm{AH} 7$ & -5.4625 & 0.345 & 4.14 \\
\hline AH8 & 6.7275 & -1.15 & 1.495 \\
\hline AH9 & 6.7275 & -0.345 & 2.484 \\
\hline AH10 & 6.7275 & -0.92 & 3.151 \\
\hline AH11 & 10.0625 & -4.255 & 0 \\
\hline AH12 & 10.0625 & 4.255 & 0 \\
\hline AV1 & -10.0625 & 0 & \multirow[t]{2}{*}{ Top of container } \\
\hline AV2 & 10.0625 & 0 & \\
\hline
\end{tabular}

\begin{tabular}{l|r|l|l}
\hline \multicolumn{5}{l}{ (b) } \\
\hline P1 & -0.5175 & -0.299 & 0.23 \\
\hline P2 & -0.5175 & -0.345 & 1.012 \\
\hline P3 & 0.0575 & -0.46 & 2.001 \\
\hline P4 & 0.7475 & -0.345 & 2.99 \\
\hline P5 & -7.8315 & 1.265 & 2.99 \\
\hline P6 & -6.3825 & 0.115 & 4.002 \\
\hline P7 & 6.4975 & 0.782 & 2.024 \\
\hline P8 & 6.7275 & 0.851 & 2.99 \\
\hline P9 & -8.0615 & -0.805 & 0.276 \\
\hline P10 & 6.7965 & -0.046 & 0.23 \\
\hline
\end{tabular}

\subsubsection{Centrifuge Equipment}

The geotechnical centrifuge at RPI has a radius of $3 \mathrm{~m}$ and can withstand a total model weight of $150 \mathrm{~g}$ tons. The shaking table is installed within the centrifuge basket and can replicate dynamic (earthquake and artificial) motions in one or two lateral directions.

\subsection{Recorded Response}

\subsubsection{Input Motion}

The sloping deposit model was subjected to two destructive excitations along the longitudinal direction. The excitations consisted of two sinusoidal ramped motions of 16 cycles, with a predominant frequency of $1 \mathrm{~Hz}$. The sequence of shaking had an 
Table 19.2 Locations of tracking targets: (a) central rows along the slope and (b) central rows across the slope

\begin{tabular}{l|l|l|l}
\hline Target row & $x(\mathrm{~m})$ & $y(\mathrm{~m})$ & $z(\mathrm{~m})$ \\
\hline (a) & -66.125 & -1.15 & 48.07 \\
\hline & -54.625 & -1.15 & 46.92 \\
\hline & -43.125 & -1.15 & 46.00 \\
\hline $\mathrm{A}$ & -31.625 & -1.15 & 45.08 \\
\hline $\mathrm{B}$ & -20.125 & -1.15 & 43.93 \\
\hline $\mathrm{C}$ & -8.625 & -1.15 & 43.01 \\
\hline $\mathrm{D}$ & 2.875 & -1.15 & 42.09 \\
\hline $\mathrm{E}$ & 14.375 & -1.15 & 41.17 \\
\hline $\mathrm{F}$ & 25.875 & -1.15 & 40.02 \\
\hline $\mathrm{G}$ & 37.375 & -1.15 & 39.1 \\
\hline $\mathrm{H}$ & 48.875 & -1.15 & 37.95 \\
\hline $\mathrm{I}$ & 60.375 & -1.15 & 36.57 \\
\hline & 71.875 & -1.15 & 35.42 \\
\hline & & &
\end{tabular}

\begin{tabular}{|c|c|c|c|}
\hline \multicolumn{4}{|l|}{ (b) } \\
\hline-3 & 2.875 & -35.65 & 41.17 \\
\hline-2 & 2.875 & -24.15 & 39.79 \\
\hline-1 & 2.875 & -12.65 & 40.25 \\
\hline 0 & 2.875 & -1.15 & 40.02 \\
\hline 1 & 2.875 & 10.35 & 39.10 \\
\hline 2 & 2.875 & 21.85 & 39.10 \\
\hline 3 & 2.875 & 33.35 & 39.10 \\
\hline
\end{tabular}

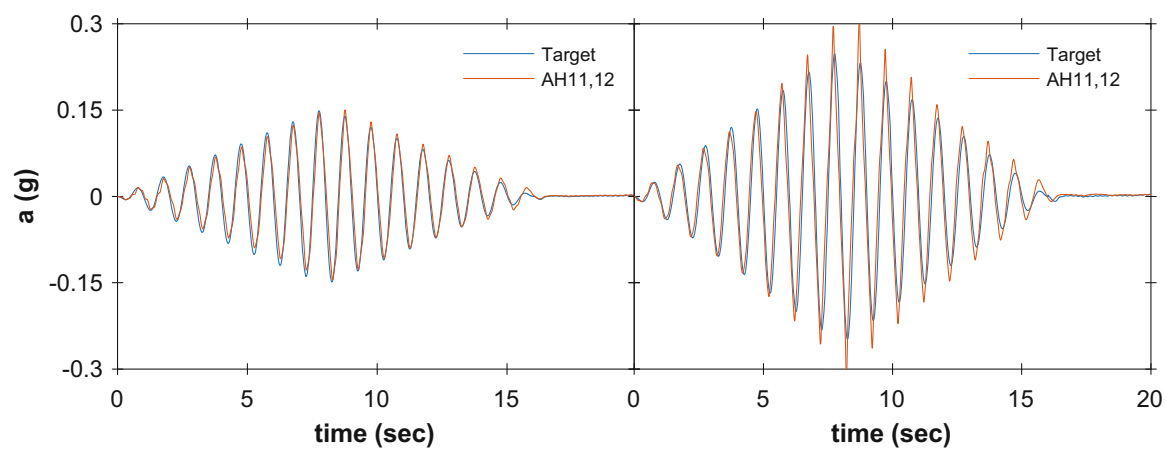

Fig. 19.6 Comparison of the target and recorded input accelerations for the 0.15 and $0.25 \mathrm{~g}$ motions of 2017_RPI01. Left-side view refers to $0.15 \mathrm{~g}$ shake and right-side view to $0.25 \mathrm{~g}$ shake

increasing intensity, with the motions having a maximum acceleration of $0.15 \mathrm{~g}$ and then $0.25 \mathrm{~g}$. Figure 19.6 shows a comparison between the target and achieved input motions and shows a remarkable level of fidelity in replicating the target in terms of both amplitude and frequency. Specifically, there is only a slight discrepancy in amplitude of the largest cycles of the $0.25 \mathrm{~g}$ motion; however, the frequency content was not affected. A non-destructive tremor was applied before and after each shake 


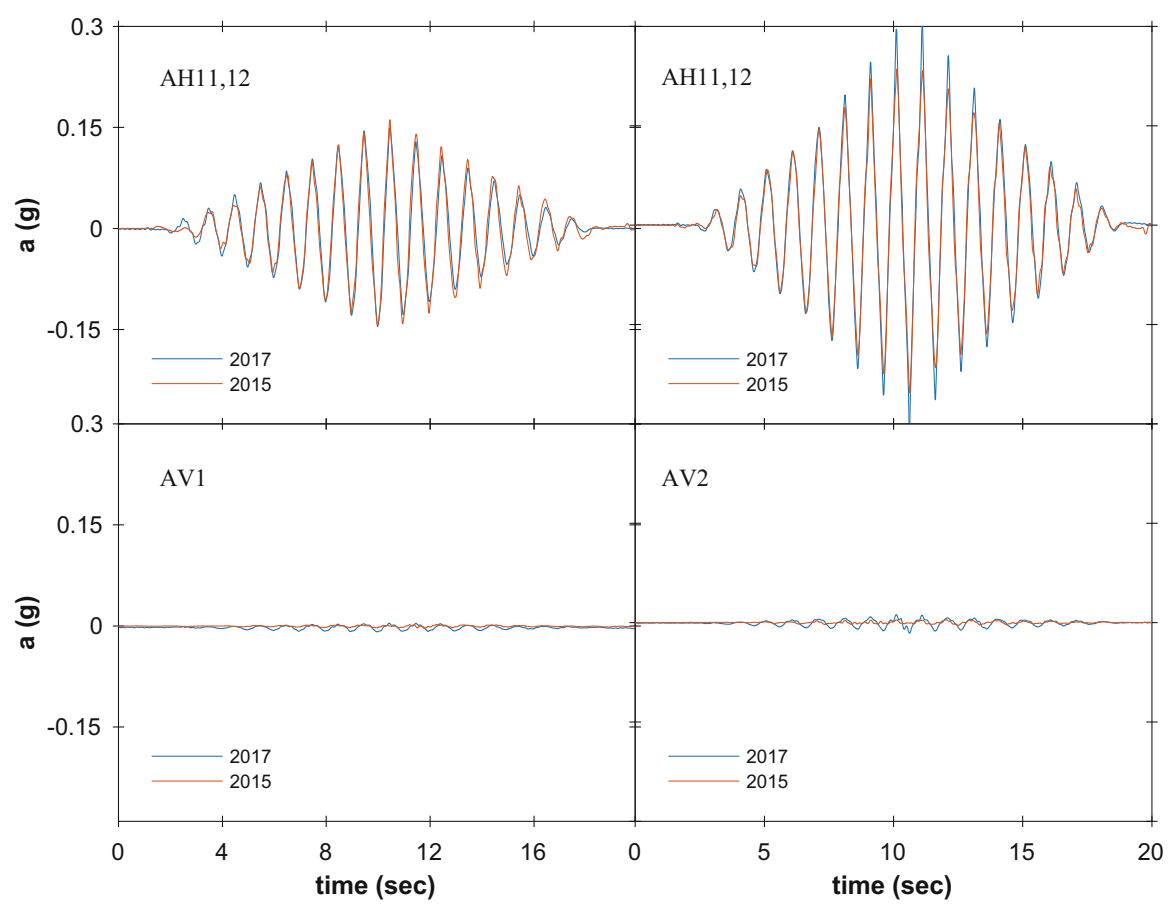

Fig. 19.7 Comparison of the recorded horizontal and vertical input accelerations of the rigid container for the 2017_RPI01 and 2015_RPI01 tests. Left-side view refers to $0.15 \mathrm{~g}$ shake and right-side view to $0.25 \mathrm{~g}$ shake

for system identification purposes. The response associated with these tremors is not included in this article for reasons of brevity.

A comparison of the input motions that were recorded by the accelerometers AH11 and AH12 (mounted at the base of the rigid container) during the 2015_RPI01 and 2017_ RPI01 tests confirmed the successful repeatability of input motion for the $0.15 \mathrm{~g}$ and $0.25 \mathrm{~g}$ shakes (Fig. 19.7). Note also that the recorded vertical accelerations are negligible from a practical point of view (for both tests), assuring that no significant rocking had occurred.

\subsubsection{Soil Acceleration}

The accelerations of the soil deposit were recorded for the 0.15 and $0.25 \mathrm{~g}$ excitations of the 2015_RPI01 and 2017_RPI01 tests at the AH1 to AH4 locations (Fig. 19.8). The sensor AH2 malfunctioned during the second excitation of the 2017_ RPI01 test. There is a remarkable agreement between the accelerations of 

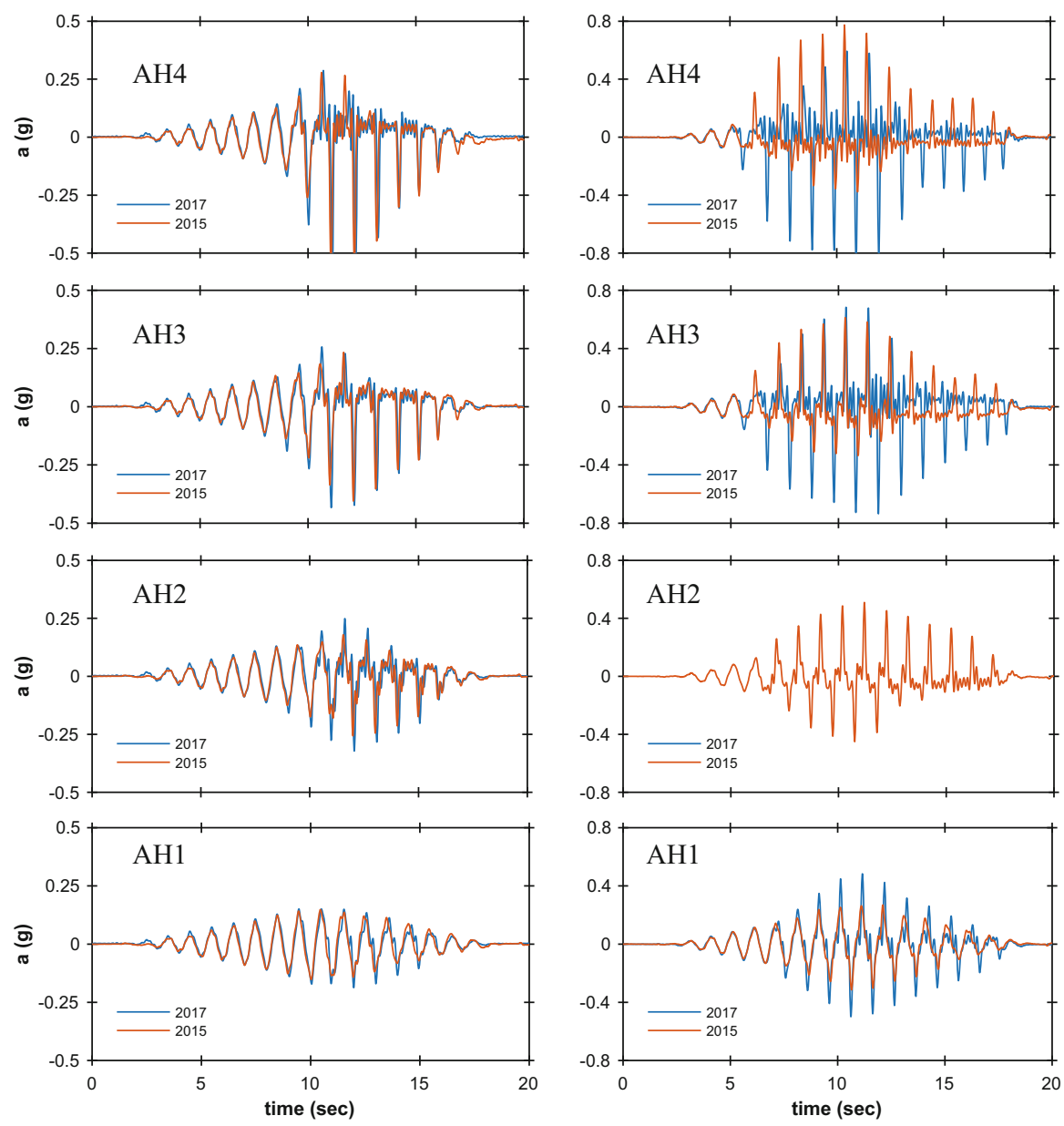

Fig. 19.8 Acceleration time histories recorded along the sensors central array during the 2017_RPI01 and 2015_RPI01 tests. Left-side view refers to $0.15 \mathrm{~g}$ shake and right-side view to $0.25 \mathrm{~g}$ shake

the two tests in terms of both frequency content and intensity of the cyclic motion, including the spikes. This is especially the case for the $0.15 \mathrm{~g}$ excitation. However, the accelerations of 2017_ RPI01 during the 0.25 g excitation were higher than those of 2015_RPI01, especially within the shallow soil layers and for the prominent negative acceleration spikes. This is attributed partly to the larger amplitude of the 2017_ RPI01 input motion for the 7-13 s cycles. The larger negative acceleration spikes are indicative of a more intense dilation that occurred in the downslope direction at high strains of the 2017_ RPI01 test, as expected. 


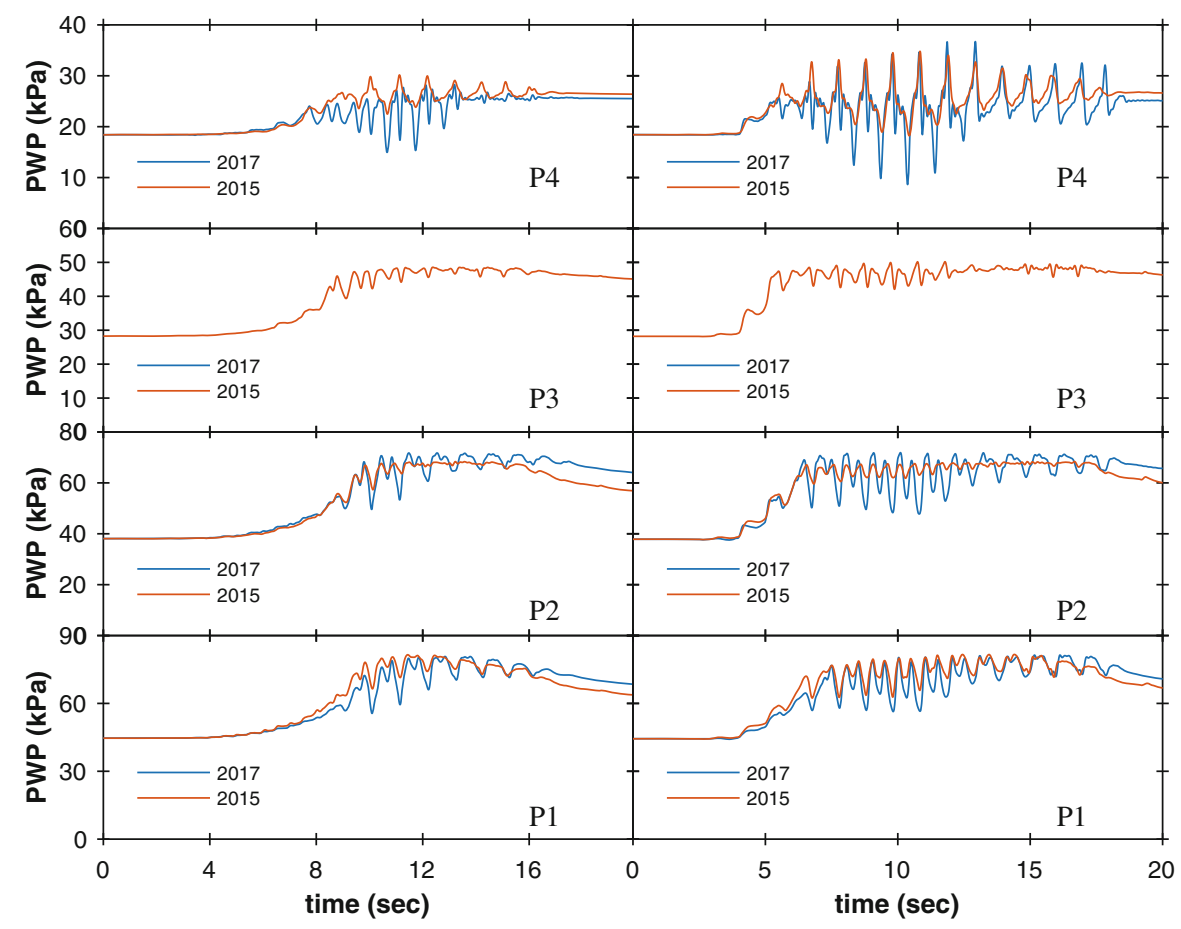

Fig. 19.9 Pore water pressure (PWP) time histories recorded along the central array during the 2017_RPI01 and 2015_RPI01 tests. Left-side view refers to $0.15 \mathrm{~g}$ shake and right-side view to $0.25 \mathrm{~g}$ shake

\subsubsection{Pore Water Pressure}

The short-term pore water pressure time histories also showed a remarkable agreement between the 2015_RPI01 and 2017_RPI01 results, as depicted in Fig. 19.9 (note that the sensor P3 malfunctioned during the 2017 test). In general, the agreement is better for the lower layers of the deposit, while closer to the surface the agreement was affected by the dilative response (in consistency with the acceleration response discussed above). The dilative response was more prevalent for the 2017_RPI01 test, especially during the strongest phase of the $0.25 \mathrm{~g}$ shaking. Similar responses were obtained for the long-term pore water pressure time histories (Fig. 19.10). The pressure dissipation rates were noticeably close, and indicate that the achieved permeability and soil fabric were consistent for the two tests. The pore pressure results are also presented in terms of excess pore water pressure ratio in Fig. 19.11. The ratio was calculated as follows:

$$
R u=\frac{u-u_{\mathrm{hydr}}}{\sigma_{v}^{\prime}}
$$




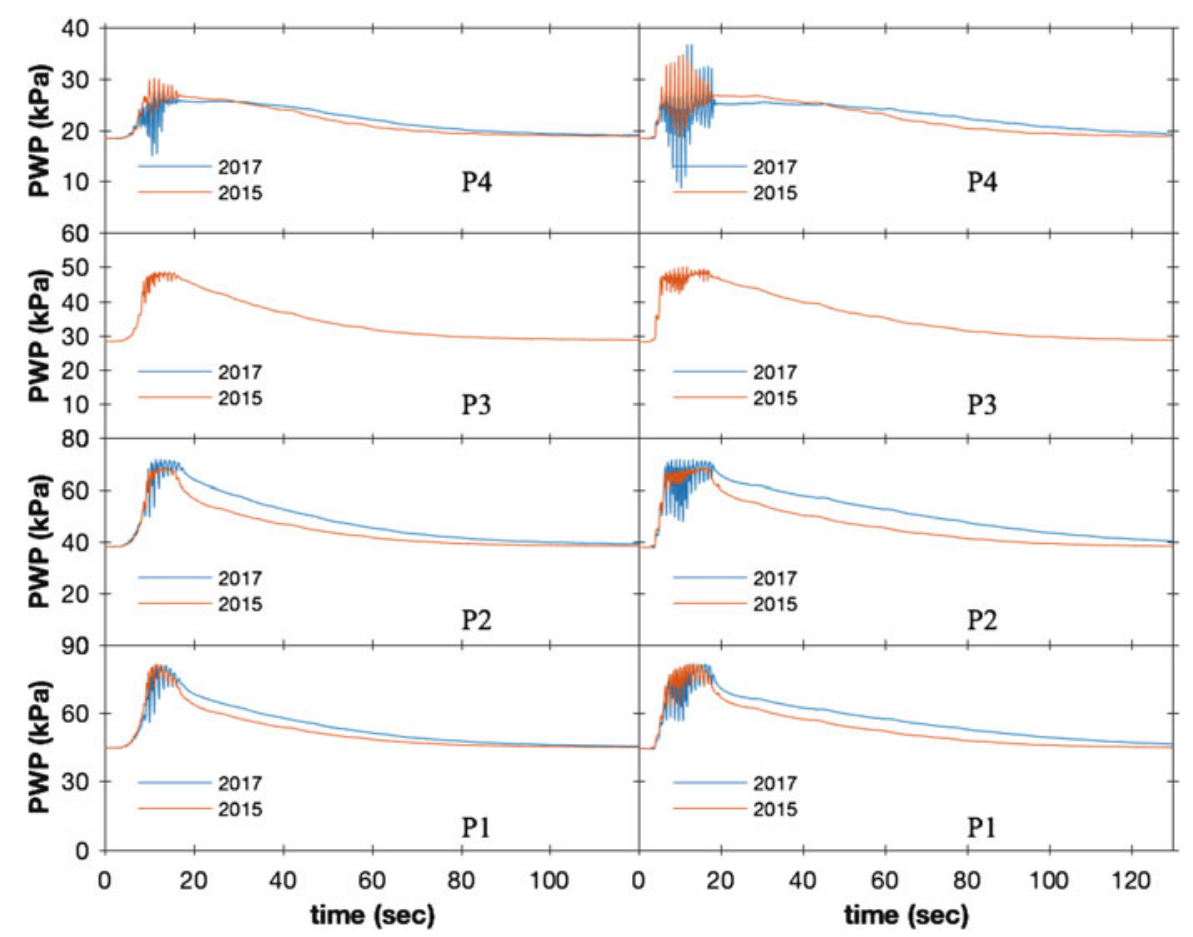

Fig. 19.10 Long-term time histories of the pore pressures recorded along the central array during the 2017_RPI01 and 2015_RPI01 tests. Left-side view refers to $0.15 \mathrm{~g}$ shake and right-side view to $0.25 \mathrm{~g}$ shake

where $u$ is the excess pore water pressure, $u_{\text {hydr }}$ is the initial hydrostatic water pressure, and $\sigma_{v}^{\prime}$ is the initial effective stress at the level of each pore water pressure transducer. The values of the $R u$ ratio varied between 1 and 0 , and that peak values of $R u$ were close to 1.0 for the entire stratum in both tests.

\subsection{Lateral Spreading}

The permanent lateral (spreading) displacements along the slope were measured by means of a Phantom v5.1 HI-G high-speed camera, produced by Vision Research. The recorded videos had an actual sampling rate of 1000 frames/s, which corresponds to 43.5 frames/s in prototype scale (i.e., a Nyquest frequency of $21.7 \mathrm{~Hz}$ ). The high-speed camera records at a resolution of $1024 \times 1024$ pixels, which corresponds to approximately $0.50 \mathrm{~mm}$ resolution in measured prototype-scale displacements at a $23 \mathrm{~g}$ centrifugal level (for the conducted tests). A more detailed description of the properties of the equipment is provided by Kokkali et al. (2018). The grid of the zip-tie heads, installed as targets on the surface of the sloping ground (Fig. 19.12), was created with a spacing of $1.15 \mathrm{~m} \times 1.15 \mathrm{~m}$, observing the 


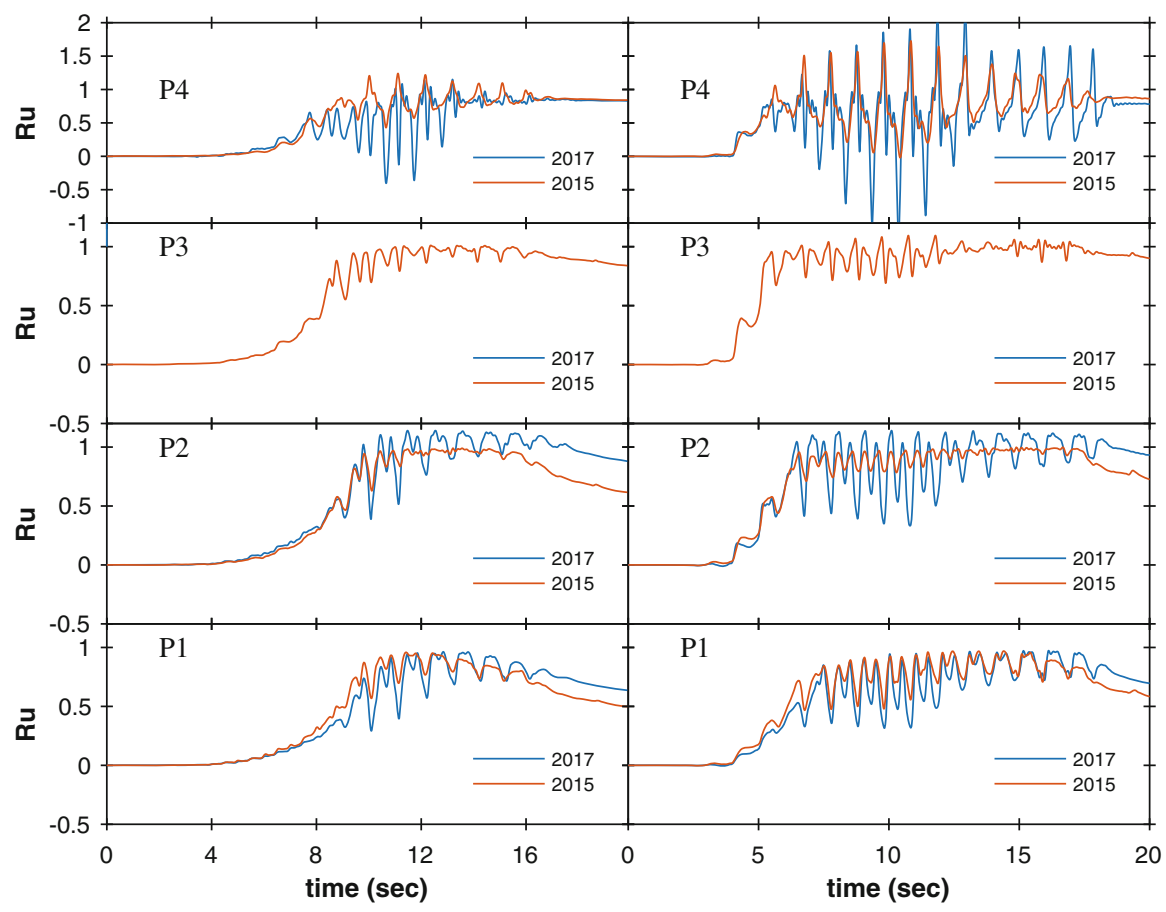

Fig. 19.11 Time histories of the excess pore pressure ratios recorded along the central array during the 2017_RPI01 and 2015_RPI01 tests. Left-side view refers to $0.15 \mathrm{~g}$ shake and right-side view to $0.25 \mathrm{~g}$ shake
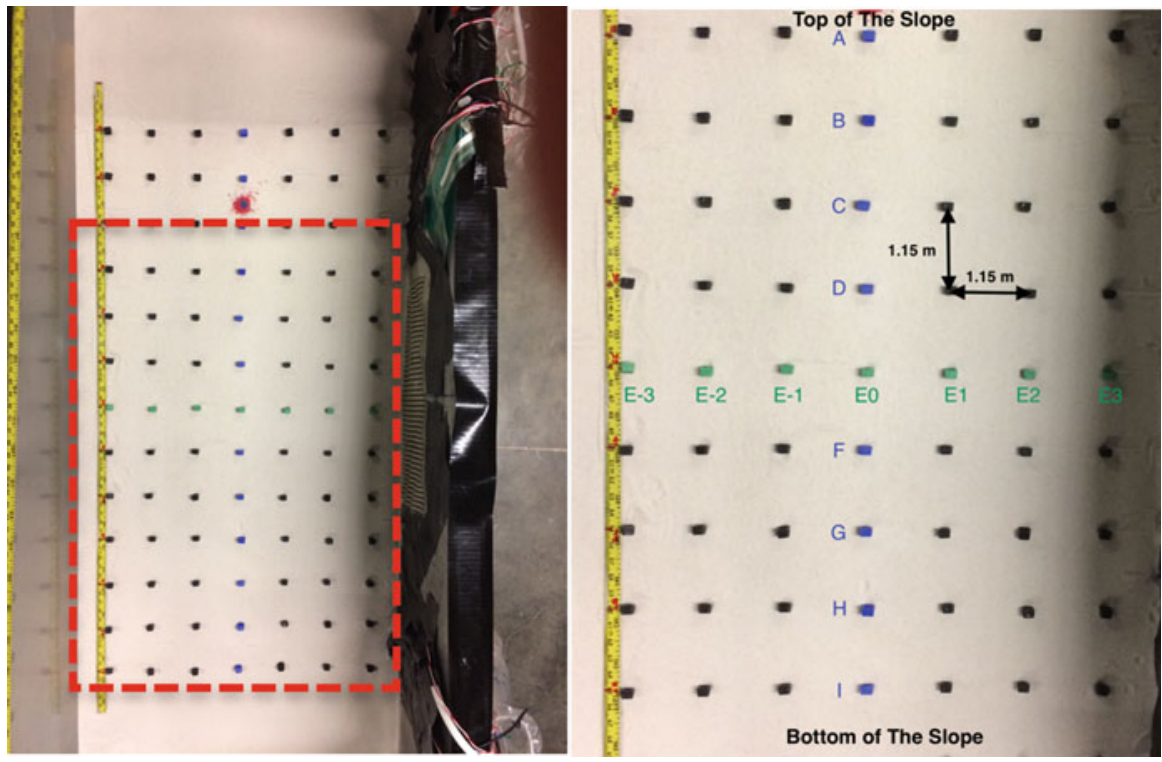

Fig. 19.12 The grid of zip-tie heads used as targets for high-speed camera tracking 


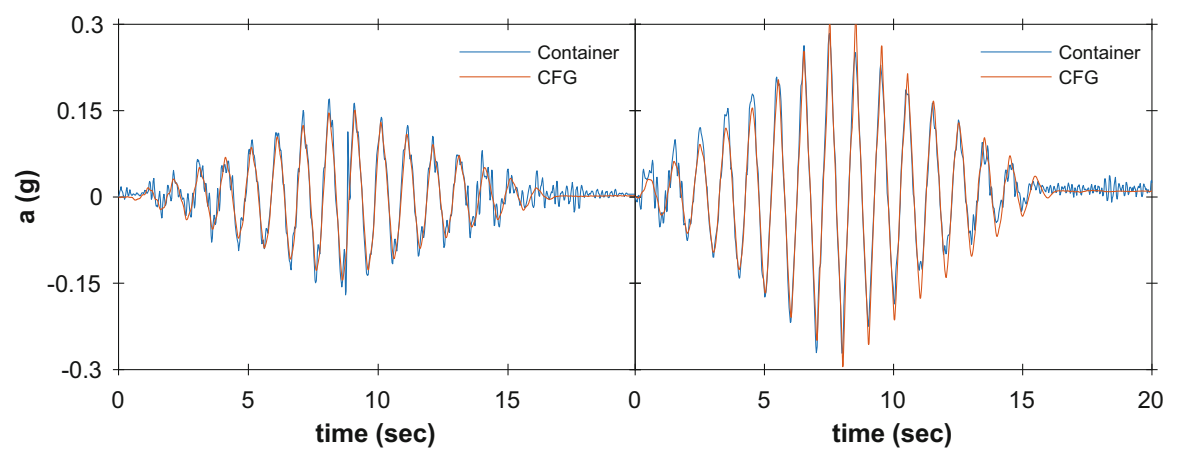

Fig. 19.13 Acceleration time histories of the input motion obtained from image analysis of highspeed camera tracking and recorded by the accelerometers AH11 and AH12. Left-side view refers to $0.15 \mathrm{~g}$ shake and right-side view to $0.25 \mathrm{~g}$ shake

methodology adopted in 2015_RPI01. The red-dashed frame in Fig. 19.12 indicates the area that was actually tracked by the high-speed camera. Some target rows were not included because of light that is reflected on waves generated within the water above the slope making it difficult to track these targets. An image analysis was used to obtain the time histories of the soil total and relative displacements. The relative displacements are evaluated by subtracting the container displacement from the soil displacement provided by processing the zip-tie head images. A double differentiation was employed to obtain the corresponding acceleration time histories. Two (target) points were tracked on the container walls to verify the quality of obtained accelerations. The accelerations evaluated from these points were compared to the input recorded by the two accelerometers $\mathrm{AH} 11$ and $\mathrm{AH} 12$ at the base of the rigid container, as shown in Fig. 19.13. This figure shows that the magnitude and frequency content of the container target acceleration is consistent with the motions measured by the accelerometers. The agreement also confirms that the container behaves practically as rigid and apparently does not affect the input motion. Further verification of the tracking and image analysis was performed by comparing the acceleration AH4 (along the central array and the closest to the slope surface) with the target D0 (at the central target of the grid in Fig. 19.12), as shown in Fig. 19.14. The time histories for AH4 and D0 were very close and practically identical for both motions. The highly dilative spikes after full liquefaction (i.e., after about $8.0 \mathrm{~s}$ ) are fully captured by target tracking. The displacement time histories for the central three target rows along the slope are exhibited in Fig. 19.15 for the 0.15 and $0.25 \mathrm{~g}$ excitation during the 2015_RPI01 and 2017_RPI01 experiments. The two tests were in remarkable agreement, showing a consistent value of permanent displacement approximately $12 \mathrm{~cm}$ in prototype scale at the end of shaking. Note that the displacements in Fig. 19.15 are shown in dotted lines after the $16 \mathrm{~s}$ instant to indicate a lower accuracy for these displacements (waves were generated within the water above the slope free surface and became more prominent after the end of shaking 


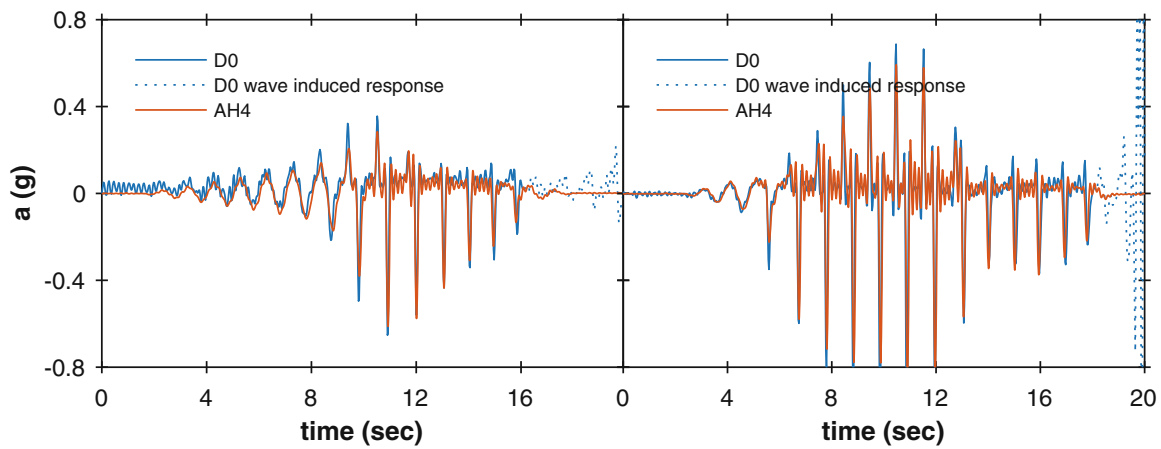

Fig. 19.14 Acceleration time histories of the motion obtained from image analysis of the central target D0 and recorded by accelerometers AH4 (closest sensor to the surface along the central array). Left-side view refers to $0.15 \mathrm{~g}$ shake and right-side view to $0.25 \mathrm{~g}$ shake
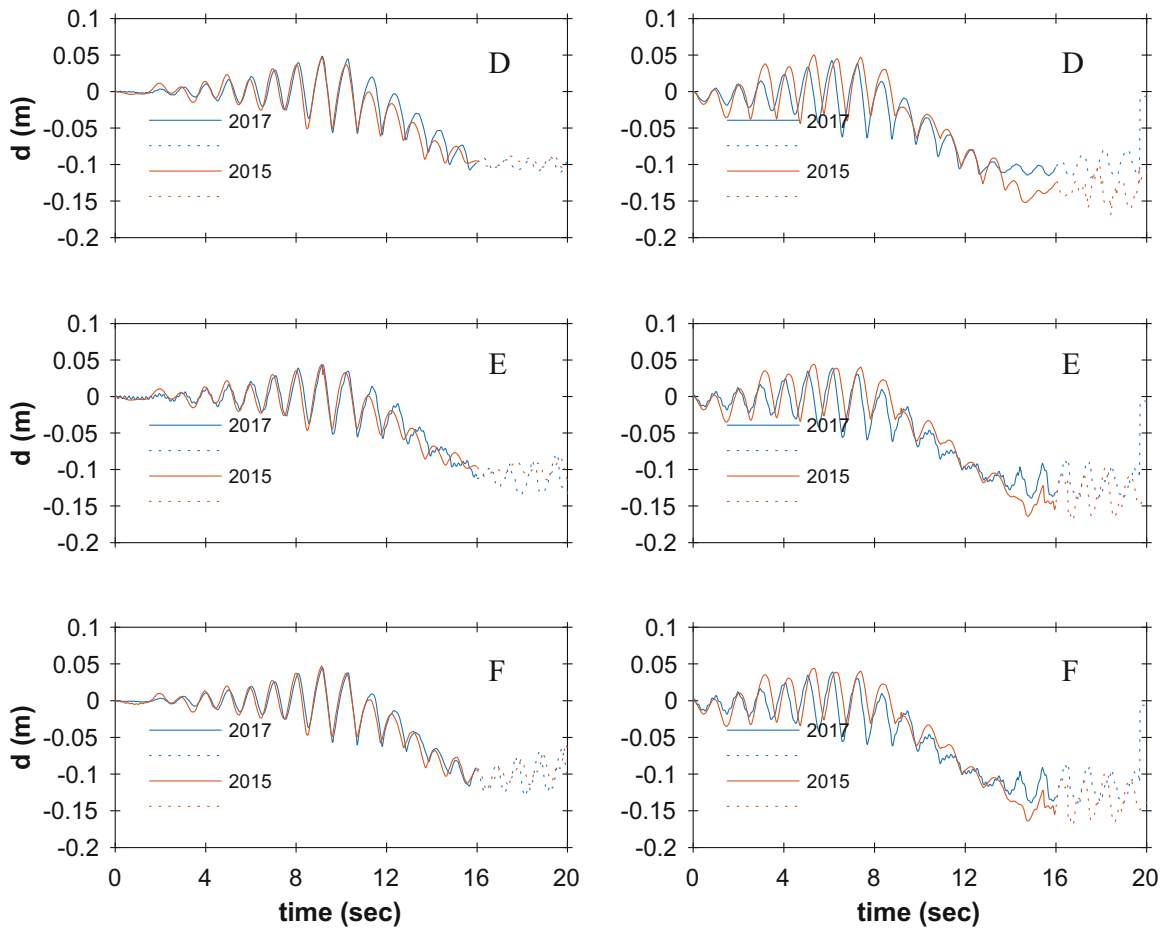

Fig. 19.15 Time histories of lateral spreading displacements along the three central rows D, E, and $\mathrm{F}$ along the slope for the $0.15 \mathrm{~g}$ (left) and $0.25 \mathrm{~g}$ (right) shakes (the dotted portion of the graphs corresponds to lower accuracy due to water wave reflections) 
which compromised the accuracy of the tracking process). Nevertheless, the overall trend of the permanent lateral displacement components remained consistent for the two tests.

\subsection{Conclusions}

Five LEAP centrifuge experiments were conducted at Rensselaer Polytechnic Institute in 2015 and 2017. This article presented, compared, and assessed the level of repeatability of two tests performed according to the same specification by two different researchers: the first test was conducted in 2015 (2015_RPI01) and the second was a repeat carried out in 2017 (2017_RPI01). Each test included a 0.15 and $0.25 \mathrm{~g}$ input excitations. The input motions were found to be highly repeatable and were consistent in frequency content and amplitude (especially for the $0.15 \mathrm{~g}$ motion) and showed that the two tests were subjected to practically the same loading conditions. The 2017 model building, instrumentation, preparation of the viscous pore fluid, and model saturation were performed following methodology of the 2015 test. The recorded accelerations and excess pore water pressures (of the two tests) were shown to be in very good agreement during the early phase of shaking and also during liquefaction and dilative response (including consistency in acceleration spikes and drops in pore pressure). The consistency in the pore pressure build-up between the two tests shows that the two models had comparable compressibility values. This finding along with the long-term time history of pore pressure dissipation confirmed that the two models have practically the same soil permeability. The lateral spreading displacements were also highly consistent not only in terms of the ultimate final values but also in time history of cyclic response. All these agreements are strong indicators that the two sloping soil deposits had consistent soil characteristics including stiffness and fabric.

Acknowledgments The authors would like to acknowledge the financial support of the National Science Foundation Geotechnical Engineering Program directed by Dr. Richard Fragaszy (Grant No. CMMI- 1635040). This support is gratefully acknowledged. The authors would also like to acknowledge the contribution of the staff of the Centrifuge Center at Rensselaer to the performance of the centrifuge tests.

\section{References}

Abdoun, T., Kokkali, P., \& Zeghal, M. (2018). Physical modeling of soil liquefaction: Repeatability of centrifuge experimentation at RPI. Geotechnical Testing Journal, 41(1), 141-163. https://doi. org/10.1520/GTJ20160192.

Carey, T. J., Stone, N., \& Kutter, B. L. (2019). Grain size analysis and maximum and minimum dry density of Ottawa F-65 sand for LEAP-UCD-2017. In B. Kutter et al. (Eds.), Model tests and 
numerical simulations of liquefaction and lateral spreading: LEAP-UCD-2017. New York: Springer.

Kokkali, P., Abdoun, T., \& Zeghal, M. (2018). Physical modeling of soil liquefaction: Overview of LEAP production test 1 at Rensselaer Polytechnic Institute. Soil Dynamics and Earthquake Engineering, 113, 629-649.

Kutter, B., Carey, T., Hashimoto, T., Zeghal, M., Abdoun, T., Kokalli, P., Madabhushi, G., Haigh, S., Hung, W.-Y., Lee, C.-J., Iai, S., Tobita, T., Zhou, Y. G., Chen, Y., \& Manzari, M. T. (2017). LEAP-GWU-2015 experiment specifications, results, and comparisons. International Journal of Soil Dynamics and Earthquake Engineering. https://doi.org/10.1016/j.soildyn.2017.05.018.

Kutter, B. L., Carey, T. J., Stone, N., Bonab, M. H., Manzari, M., Zeghal, M., Escoffier, S., Haigh, S., Madabhushi, G., Hung, W., Kim, D., Kim, N., Okamura, M., Tobita, T., Ueda, K., \& Zhou, Y. (2019). LEAP-UCD-2017 V. 1.01 model specifications. In B. Kutter et al. (Eds.), Model tests and numerical simulations of liquefaction and lateral spreading: LEAP-UCD-2017. New York: Springer.

Manzari, M., Ghoraiby, M. E., Kutter, B. L., Zeghal, M., Abdoun, T., Arduino, P., Armstrong, R. J., Beaty, M., Carey, T., Chen, Y.-M., Ghofrani, A., Gutierrez, D., Goswami, M., Haigh, S. K., Hung, W.-Y., Iai, S., Kokkali, P., Lee, C.-J., Madabhushi, S. P. G., Mejia, L., Sharp, M., Tobita, T., Ueda, K., Zhou, Y.-G., \& Ziotopoulou, K. (2018). Liquefaction analysis and experiment projects (LEAP): Summary of observations from the planning phase. International Journal of Soil Dynamics and Earthquake Engineering, 113, 714-743. https://doi.org/10.1016/j.soildyn. 2017.05.015.

Open Access This chapter is licensed under the terms of the Creative Commons Attribution 4.0 International License (http://creativecommons.org/licenses/by/4.0/), which permits use, sharing, adaptation, distribution and reproduction in any medium or format, as long as you give appropriate credit to the original author(s) and the source, provide a link to the Creative Commons license and indicate if changes were made.

The images or other third party material in this chapter are included in the chapter's Creative Commons license, unless indicated otherwise in a credit line to the material. If material is not included in the chapter's Creative Commons license and your intended use is not permitted by statutory regulation or exceeds the permitted use, you will need to obtain permission directly from the copyright holder.

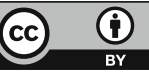

